7th International Workshop on Astronomy and

Relativistic Astrophysics (IWARA 2016)

International Journal of Modern Physics: Conference Series

Vol. 45 (2017) 1760050 (4 pages)

(C) The Author(s)

DOI: $10.1142 / \mathrm{S} 2010194517600503$

\title{
Equatorial Geodesics Around the Magnetars
}

\author{
Viviane A. P. Alfradique, Orlenys N. Troconis, and Rodrigo P. Negreiros \\ Departmento de Física, Universidade Federal Fluminense (UFF) \\ 24210-346 Niterói, Rio de Janeiro, Brazil \\ vivianeapa@if.uff.br, troconiso@if.uff.br,negreiros@if.uff.br
}

Published 15 August 2017

\begin{abstract}
Neutron stars manifest themselves as different classes of astrophysical sources that are associated to distinct phenomenology. Here we focus our attention on magnetars (or strongly magnetized neutron stars) that are associated to Soft Gamma Repeaters and Anomalous X-ray Pulsars. The magnetic field on surface of these objects, reaches values greater than $10^{15} \mathrm{G}$. Under intense magnetic fields, relativistic effects begin to be decisive for the definition of the structure and evolution of these objects. We are tempted to question ourselves to how strengths fields affect the structure of neutron star. In this work, our objective is study and compare two solutions of Einstein-Maxwell equations: the Bonnor solution, which is an analytical solution that describe the exterior spacetime for a massive compact object which has a magnetic field that is characterize as a dipole field and a complete solution that describe the interior and exterior spacetime for the same source found by numerical methods). For this, we describe the geodesic equations generated by such solutions. Our results show that the orbits generated by the Bonnor solution are the same as described by numerical solution. Also, show that the inclusion of magnetic fields with values up to $10^{17} \mathrm{G}$ in the center of the star does not modify sharply the particle orbits described around this star, so the use of Schwarzschild solution for the description of these orbits is a reasonable approximation.
\end{abstract}

Keywords: Magnetars, General Relativity, Geodesics.

PACS numbers: 04.25D, 04.20.Jb, 04.40Nr, 97.60.Jd

\section{Introduction}

In scope of high-energy astrophysics, magnetic fields are studied in white dwarfs, neutron stars, pulsars and black holes. The intensity of the magnetic field varies according to the astrophysical object being studied. Magnetars are neutron stars highly magnetized, the magnetic fields present on the object surfaces are of the order of $10^{14}-10^{15} \mathrm{G}$ and can reach even greater values in its center; this makes them the strongest magnets in the present universe since the combination of the

This is an Open Access article published by World Scientific Publishing Company. It is distributed under the terms of the Creative Commons Attribution 4.0 (CC-BY) License. Further distribution of this work is permitted, provided the original work is properly cited. 
extreme density and magnetic field put them in the strong field limit. The idea of this kind of astrophysical object was suggest in an attempt to understand the large secular spin-down rate and the long spin periods (clustered in the range of $\approx 2-12 s$. The main candidate of magnetars are the soft-gamma repeaters and the Anomalous X-ray pulsars that agreed with the magnetar scenario. Duncan and Thompson ${ }^{1-3}$ were the first to try to explain this by proposing that the decay of a super-strong magnetic field supports this high observed values of secular spin-down rate and spin periods.

In this astrophysical ambient form by massive objects and with an intensive magnetic field, the use of the general relativity is essential for the description of the structure of these objects. In this work we will present the equations of geodesics that describe the movement of particles around a compact object with a dipole magnetic field in two distinct approaches, the first one an analytical approach built from the solution describe by Bonnor ${ }^{4}$, but removing the description of the electric field; and the second a numerical approach developed from the LORENE code, known in the literature ${ }^{5,6}$, which makes use of pseudo-spectral numerical technique.

\section{Geodesic Equations in the Equatorial Plane}

The metric that describe the exterior gravitational field of a mass possessing a magnetic dipole moment was found by Bonnor ${ }^{4}$ and can be written as

$$
\begin{aligned}
d s^{2} & =-\left(1-\frac{2 m}{r}+\frac{\mu^{2} \cos ^{2} \theta}{r^{4}}\right) d t^{2}+\left(1-\frac{2 m}{r}+\frac{\mu^{2} f(\theta)}{r^{4}}\right)^{-1} d r^{2} \\
& +r^{2}\left(1-\frac{\mu^{2} f(\theta)}{r^{4}}\right) d \theta^{2}+r^{2} \sin ^{2} \theta\left(1-\frac{\mu^{2} \cos ^{2} \theta}{r^{4}}\right) d \alpha^{2}
\end{aligned}
$$

where terms of higher order than 2 in the gravitational constant, mass $m$, and magnetic dipole $\mu$ were excluded. Electromagnetic source is given by the 4-potential $A^{\mu}=\left(0,0,0, \frac{\mu \sin ^{2} \theta}{r}\right)$. A general metric that describe a static axially symmetric solution is written in the form ${ }^{5}$

$$
d s^{2}=-N^{2} d t^{2}+A^{2}\left(d r^{2}+r^{2} d \theta^{2}\right)+B^{2} r^{2} \sin ^{2} \theta d \phi^{2},
$$

where $B, A$ and $N$ are functions of $r$ and $\theta$. The geodesics equations are found from relativistic Euler-Lagrange equations, this equations provide the conservations of the two quantities due to the symmetry imposed on the system (the solution is static and axially symmetric). The particle orbit equation for the solutions describe above was found from the equations of geodesics in the equatorial plane $\left(\theta=\frac{\pi}{2}\right)$

$$
\frac{d R}{d \phi}= \pm \frac{g_{\phi \phi}\left(r, \frac{\pi}{2}\right)}{\ell_{e q}} \sqrt{\frac{1}{g_{r r}\left(r, \frac{\pi}{2}\right)}\left(1 \frac{E_{e q}^{2}}{g_{t t}\left(r, \frac{\pi}{2}\right)}-\frac{\ell_{e q}^{2}}{g_{\phi \phi}\left(r, \frac{\pi}{2}\right)}\right)}
$$

where $E_{e q}$ is the conserved energy per unit rest mass and $\ell_{e q}$ is the conserved angular momentum per unit rest mass. In Figs. 1-2 we graph the solutions of the 

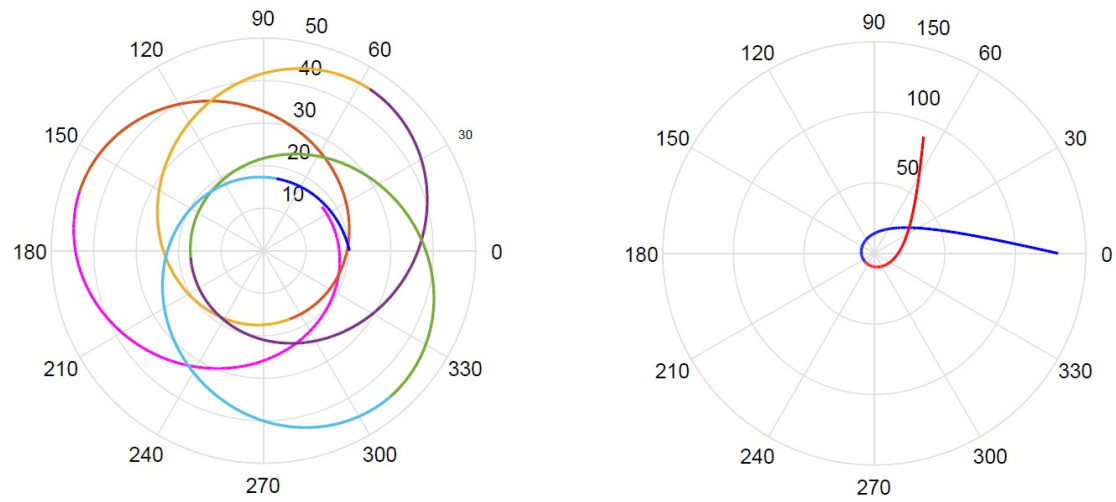

Fig. 1. Orbit of a test particle in a spacetime described by Bonnor solution. The values of the parameters used are $\ell_{e q}=8.557, \mu=2.078 \mathrm{~km}^{2}$ and $m=1.99 M_{\odot}$.
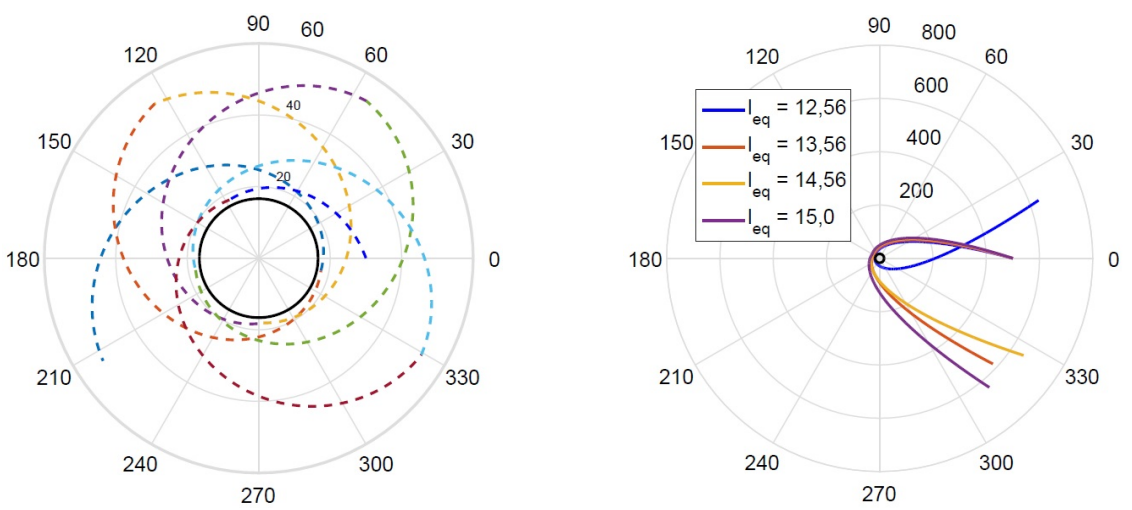

Fig. 2. Orbit of a test particle in a spacetime described by the numeric solution found by using the LORENE code. The values of the parameters used are $\mu=2.15 \mathrm{~km}^{2}, m=1.899 M_{\odot}$ and $R=16.61 \mathrm{~km}$. The black solid line represent the radius of the star that was calculated using a polytropic equation of state with the following parameters $\gamma=0.05$ and $\kappa=0.05 \frac{\rho_{n u c l} c^{2}}{n_{n u c l}^{\gamma}}$.

above equation for different values of the parameters to describe different models of magnetized neutron stars and for the two distinct approach (Bonnor and the numerical solution). The effective potential responsible for generate these orbits was found for the Bonnor solution and for the numerical solution respectively:

$$
\begin{aligned}
& V e f^{B}=\frac{1}{2}\left[\left(\frac{\mu^{2}}{2 r^{4}}-\frac{2 m}{r}\right) \frac{\ell_{e q}^{2}}{r^{2}}\left(1-\frac{2 m}{r}-\frac{\mu^{2}}{2 r^{4}}\right)-\frac{\mu^{2} E_{e q}^{2}}{2 r^{4}\left(1-\frac{2 m}{r}\right)}\right], \\
& V e f^{L}=-\frac{1}{2}\left(\frac{d r}{d \tau}\right)^{2}=-\frac{1}{2 A^{2}\left(r, \frac{\pi}{2}\right)}\left(1+\frac{E_{e q}^{2}}{N^{2}\left(r, \frac{\pi}{2}\right)}-\frac{\ell_{e q}^{2}}{r^{2} B^{2}\left(r, \frac{\pi}{2}\right)}\right) .
\end{aligned}
$$



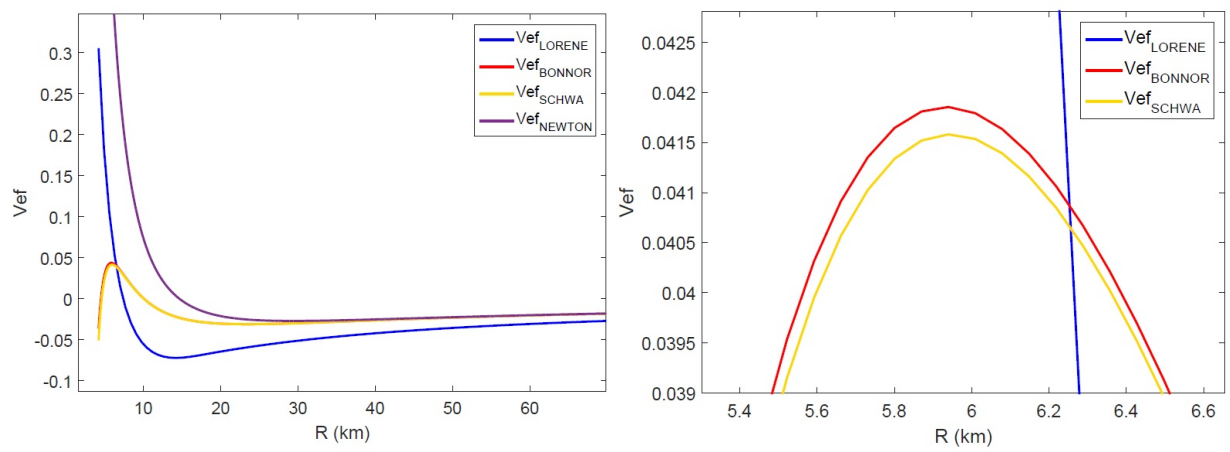

Fig. 3. Profile of the effective potential for Bonnor solution (red curve), for Schwarzschild solution (curve in yellow), for Newton solution (wine curve) and for the solution of LORENE (blue curve). The following parameter values were used: $\ell_{e q}=6.81, E_{e q}=0.02145, m=1.58 M_{\odot}$ and $\mu=$ $2.22 \mathrm{~km}^{2}$ generates a central magnetic field of $10.693 \times 10^{16} \mathrm{G}$.

\section{Conclusions}

The gist of this paper is study and compare solutions that describing the exterior spacetime of a magnetic dipole mass source $m$ from two distinct approaches: an numerical and a analytical solution. To perform such a comparison we find the orbits of the massive particles described in the spacetime defined by these solutions. Because they are physical invariants, the orbits should not be different from each other since the gravitational source is the same for both solutions.

In the light of all these results we can conclude that for central magnetic fields of the order of $10^{17} G$ the Bonnor solution describes qualitatively the orbits of massive particles around the object described by it, and the effects of insertion of the magnetic field are negligible in the description of the structure of neutron star making the use of the Schwarzschild solution is satisfactory. However quantitatively the effective potential is different of the numerical solution which shows that the Bonnor solution is not exactly the outside solution for a static massive magnetic dipole source. We also show that the only variation caused by the presence of the magnetic field is the increase of the height of the centrifugal barrier of the orbits of the massive particles (see Fig. 3).

\section{References}

1. C. Thompson and R. C. Duncan, Astrophys. J. 408, L9 (1992).

2. R. C. Duncan and C. Thompson, Astrophys. J. 408, 194 (1993).

3. C. Thompson and R. C. Duncan, Mon. Not. R. Astron. Soc. 275, 255 (1995).

4. W. B. Bonnor, Phys. Lett. A 158, 23 (1991).

5. S. Bonazzola, E. Gourgoulhon, M. Marck, M. Salgado, and J. A. Marck, Astron. Astrophys. 278, 421 (1993).

6. M. Bocquet, S. Bonazzola, E. Gourgoulhon, and J. Novak, Astron. Astrophys. 301, 757 (1995). 